\title{
CARACTERÍSTICAS EPIDEMIOLÓGICAS DE PACIENTES PEDIÁTRICOS CON DIABETES MELLITUS TIPO 1, TEGUCIGALPA-HONDURAS
}

\author{
EPIDEMIOLOGICAL CHARACTERISTICS OF PEDIATRIC PATIENTS WITH DIABETES \\ Mellitus Type 1, Tegucigalpa-HonduRAS
}

Karla Alejandra Lagos Padilla ${ }^{1}$, Sámuel Andres Barrientos Guevara ${ }^{1}$.

\section{RESUMEN}

Introducción: Dentro de la problemática del diagnóstico, tratamiento y pronóstico de patologías en infantes; la pobre educación respecto a cómo se desarrollan y el conocimiento carente en relación a las enfermedades metabólicas, conlleva a este fenómeno, al no ser identificado por padres o tutores del infante, llegando a ser factor de riesgo para el desarrollo de estas enfermedades y sus complicaciones que llegan a ser fatales.

Objetivo: Conocer la edad, género y etiología en la cual se presenta mayormente la Diabetes Mellitus Tipo I dentro de la población seleccionada. Identificar los factores predisponentes para su desarrollo, sintomatología inicial y complicaciones más frecuentes.

Metodología: Se realizó un estudio descriptivo, de corte transversal, en base a expedientes de pacientes que han sido tratados respecto a esta patología. Se consideró una población total de 51 pacientes con antecedentes de Diabetes Mellitus tipo I tomando muestra de 49 casos.

Resultados: El género femenino con 27 casos presentándose con incidencia máxima de 14 años respecto al género masculino con 22 casos con pico de edad a los 16 años.

La principal etiología fue autoinmunitario representándose en $79 \%$ de los casos. Obesidad presente en el I2\%, contrastado con pacientes que no presentaban factor de riesgo (75\%). Respecto a síntomas iniciales, la hiperfagia fue la más frecuente, seguido de polidipsia, pérdida de peso y poliuria. La principal complicación fue cetoacidosis con $35 \%$ de frecuencia

Conclusión: En la población pediátrica con Diabetes Mellitus tipo I en Honduras predomina la etiología autoinmune, desarrollandose mayormente en el género femenino.

\section{ABSTRACT}

Introduction: Inside the problematic of the diagnosis, treatment and prognosis of infant pathology, the poor education respect to how they develop and the lack of knowledge in relationship to the metabolic diseases, that entrails to this phenomenon, not being identificated by parents nor tutors of the infant, leading up as a risk factor to the development of these diseases and their complications that can be fatal.

Objective: Know the age, genre and ethiology in which Diabetes Mellitus Type I is mostly presented inside the selected population. Identify the predisposing factors for its developing, initial symptomatology and most frequent complications.

Results: The feminine gender with 27 cases presenting itself with a peak of incidence at 14 years compared with the male gender with 22 cases and a peak of incidence at 16 years.

The most frequent etiology in both genre was autoimmune, representing $79 \%$ of the cases. Obesity was present in the $12 \%$, in contrast with patients that didn't presented any risk factor (75\%). In respect of initial symptoms; hyperphagia was the most frequent, followed up by polydipsia weight loss and polyuria. The principal complication was ketoacidosis with a $35 \%$ frequency.

Conclusion: In the pediatric population with type I Diabetes Mellitus in Honduras, autoimmune etiology predominates, mostly developing in the feminine genre.

\section{INTRODUCCIÓN}

A nte la creciente incidencia de Diabetes AMellitus de Tipo 1 (DM1) en la población joven que la padece, continua la alta morbimortalidad que le acompaña ${ }^{1}$ aumentando el riesgo de sus complicaciones, pero para lograr un adecuado manejo son vitales los cambios en el estilo de vida, alimentación y la implantación de prácticas de autocuidado ${ }^{2,3}$. La guía práctica sobre DM 1 de Perú
${ }^{1}$ Médico en Servicio Social de la Universidad Nacional Autónoma de Honduras

Correspondencia a:

Karla Alejandra Lagos Padilla Correo:

karlita_lagos@hotmail.com y samuel7guevara@gmail.com

Palabras clave: Diabetes mellitus tipo 1, autoinmune, hiperfagia, obesidad cetoacidosis diabética

Keywords: Diabetes mellitus type 1 , autoimmune, hyperphagia, obesity, ketoacidosis

Procedencia y arbitraje: no comisionado, sometido a arbitraje externo.

Recibido para publicación: 26 de septiembre de 2018 Aceptado para publicación: 28 de diciembre de 2018

Citar como:

Rev Cient Cienc Med 2018;22(1): 29- 35 
lo define como "un grupo de enfermedades metabólicas caracterizadas por hiperglucemia secundaria a defectos en la secreción de insulina, que se acompaña, en mayor o menor medida, de alteraciones en el metabolismo de los lípidos y de las proteínas". En la DM1 la destrucción de las células $\beta$ del páncreas conduce a una deficiencia absoluta de insulina.

Actualmente se subdivide en dos subtipos: DM1 Inmunomediada; resultado de una destrucción autoinmune de las células $\beta$ del páncreas. Por otro lado, la DM1 Idiopática; en la cual el páncreas en incapaz de producir insulina (insulinopenia), produciendo un alto nivel de cetonas en el cuerpo que podría desencadenar una cetoacidosis en donde no se conoce el mecanismo exacto de destrucción de la célula $\beta^{5}$. Los factores genéticos modulan la intensidad y de la cronobiología de la agresión. Según el artículo "Estrategias para la prevención de la DM1": En el genoma humano la región más ligada a esta enfermedad, dentro del Sistema Mayor de Histocompatibilidad es la que codifica los genes de clase $\mathrm{II}^{6}$.

Los síntomas son atribuibles a la hiperglicemia y la evolución de esta; en concreto son: poliuria, polidipsia, polifagia, astenia y pérdida de peso, las cuales conforman la triada clásica en el niño, precedido de un período variable asintomático. En algunos casos, existe una rápida progresión de los síntomas, como: vómitos, deshidratación, y alteraciones de la conciencia ${ }^{7}$. En tiempos anteriores se han asociado los factores entre la genética $y$ la DM1, también es importante reconocer los desencadenantes ambientales que llegan a inducir el fenómeno de autoinmunidad hacia las células $\beta$ del páncreas, obtenidos dentro de ellos la deficiencia de vitamina $\mathrm{D}$, la temprana interacción con las proteínas de la vaca, la exposición limitadas hacia ciertos microrganismos en la infancia, el antecedente de parto vía cesárea y la comorbilidad de obesidad infantil. En los últimos años, se ha observado que el perfil de riesgo cardiovascular desfavorable, el síndrome metabólico y las características fenotípicas de los pacientes con DM1 son cada vez más similares a las de la Diabetes Mellitus de Tipo 2 (DM2) ${ }^{8,9}$. En la DM1 se encontró que las edades mayor incidencia debutan entre 10-15 años, etapa importante para el desarrollo emocional e individuación personal. Los pacientes llegar al diagnóstico desarrolla estrés en la vida de los infantes, afectando su calidad de vida de manera amplia, es aquí donde yace la importancia de la educación respetiva hacia padres y pacientes ${ }^{10,11}$.

La hipoglucemia es la complicación que genera más miedo y se debe evitar en la medida de lo posible, pero sus síntomas pueden variar al presentarse según cada persona ${ }^{12}$. Respecto a la Hiperglucemia, la Cetoacidosis Diabética (DKA) algunas veces es el primer signo de DM1 en personas que aún no han recibido el diagnóstico. Una infección, una lesión, una enfermedad seria, una cirugía o pasar por alto dosis de insulina pueden llevar a DKA en personas con $\mathrm{DM}^{13}$.

Cuantificar al cabo de cuántos años surgen las complicaciones es difícil, ya que dependen de cada persona y de su control metabólico ${ }^{13}$. Varios autores consideran al valor de $\mathrm{HbAlc}$ como un marcador de riesgo de complicaciones microvasculares y probablemente macrovasculares, mantenerla dentro de parámetros normales disminuye el riesgo de sufrir complicaciones ${ }^{14,15}$. Dentro de las demás complicaciones asociadas a la DM1 se encuentran la enfermedad bucodental, susceptibilidad a enfermedades infecciosas, alteraciones del aparato digestivo y dermopatías asociadas ${ }^{16}$.

El presente estudio, fue realizado con el fin de conocer la edad, género y etiología bajo la cual se presenta con mayor frecuencia la DM1 dentro de la población seleccionada, identificar los factores predisponentes para su desarrollo, sintomatología inicial y complicaciones más frecuentes en los pacientes del Hospital María de Especialidades Pediátricas para tener un mejor control epidemiológico de dicha patología y poder brindar una mejor atención en salud.

\section{MATERIALES Y MÉTODOS}

Se realizó un estudio Observacional, descriptivo, de corte transversal, utilizando los expedientes digitales propios de pacientes con diagnóstico de DM1 que son atendidos vía con sulta externa, del "Hospital María de Especialidades Pediátricas" en la ciudad de Tegucigalpa, Honduras, en el periodo mayo-junio del 2017. Se contó con una población de $\mathrm{N}=51$ expedientes brindados por el departamento de estadística, de los cuales se descartaron 2 por no contar con información completa sobre valores laboratoriales requeridos para este estudio. Se contó con una población de estudio de $n=49$ pacientes que cumplieron con los criterios de elegibilidad para el estudio: Información completa sobre sexo del paciente, edad, síntomas iniciales, factores predisponentes, diagnóstico etiológico, complicaciones, antecedentes familiares, Índice de Masa Corporal (IMC), glicemia al diagnóstico, Hemoglobina Glicosilada (Hb1Ac), cumplimiento del tratamiento.

La información obtenida se manejó de forma 
anónima sin otro uso aparte de esta investigación. Se trabajó con autorización por parte del Departamento de Epidemiología, en una base de datos realizada en el programa Microsoft Excel ${ }^{\circledR}$ para Windows ${ }^{\circledast}$ y se tabularon los datos en el programa estadístico Epi Info $7^{\circledast}$ para posteriormente presentar tablas y gráficos que representan la frecuencia e relación a un subgrupo de variables como ser edad, género, IMC, etiología, factores predisponentes y complicaciones que más se presentaron.

\section{RESULTADOS}

Una vez realizado el análisis de los casos se dilucidaron los siguientes datos: Del total estudiado, el sexo femenino resulto ser el más afectado con el
$55,10 \%$ ( $\mathrm{n}: 27)$, mientras que el sexo masculino fue de $45,90 \%$ (n:22).

En las niñas la incidencia máxima de DM1 fue a la edad de 14 años con 18,52\% (5 niñas de 27 en total) a diferencia de los varones la cual fue a los 16 años con $36,36 \%$ ( 8 niños de los 22 en total). (Figura 1)

La mayoría de los pacientes presentó un IMC normal con un $51 \%(\mathrm{n}: 25)$, encontrando bajo peso en 34\% (n:17) de los pacientes. (Figura2)

El orden de síntomas en frecuencia descendiente fue la hiperfagia con 36,73\% (n:18) polidipsia con $32,65 \%$ (n:16), en pérdida de peso 30,61\% (n:15) y poliuria con un $26,53 \%$ (n:13)

La etiología más frecuente (Tabla 1) en ambos sexos fue autoinmunitaria con un 79\% (n:39).

La obesidad (Tabla 2) fue el principal factor de

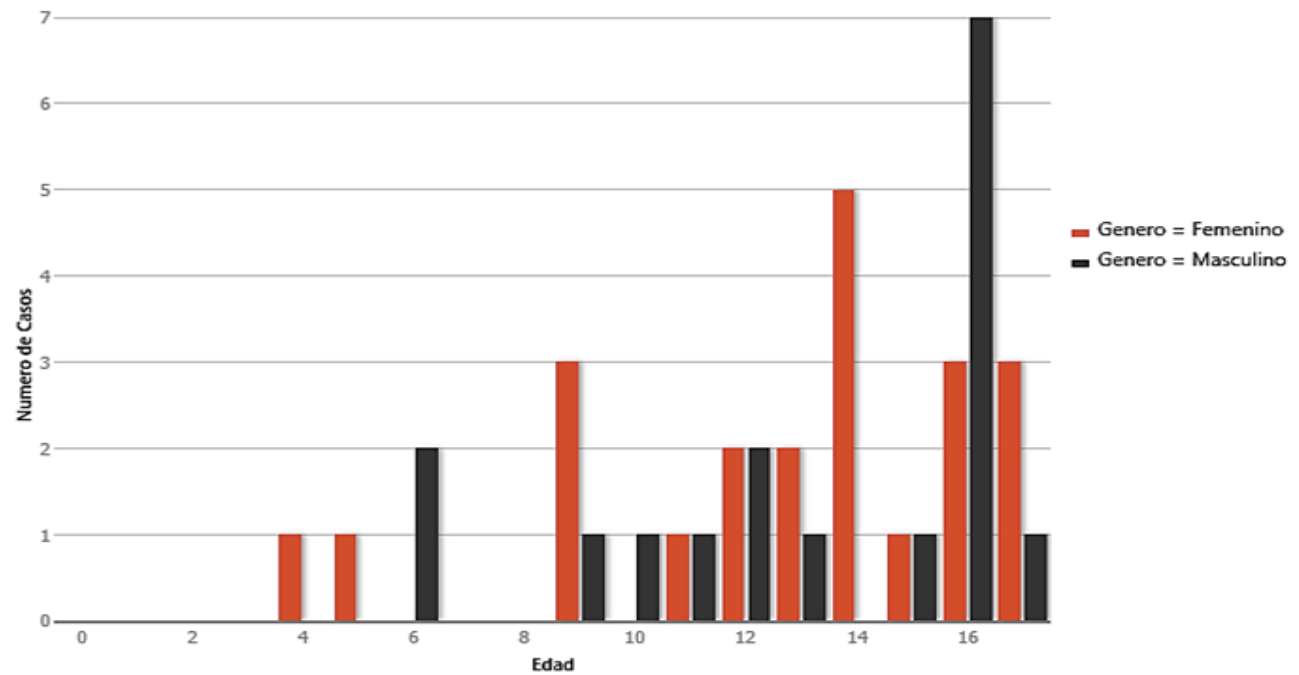

Figura 1: Incidencia de DM1 autoinmune según Edad y Género

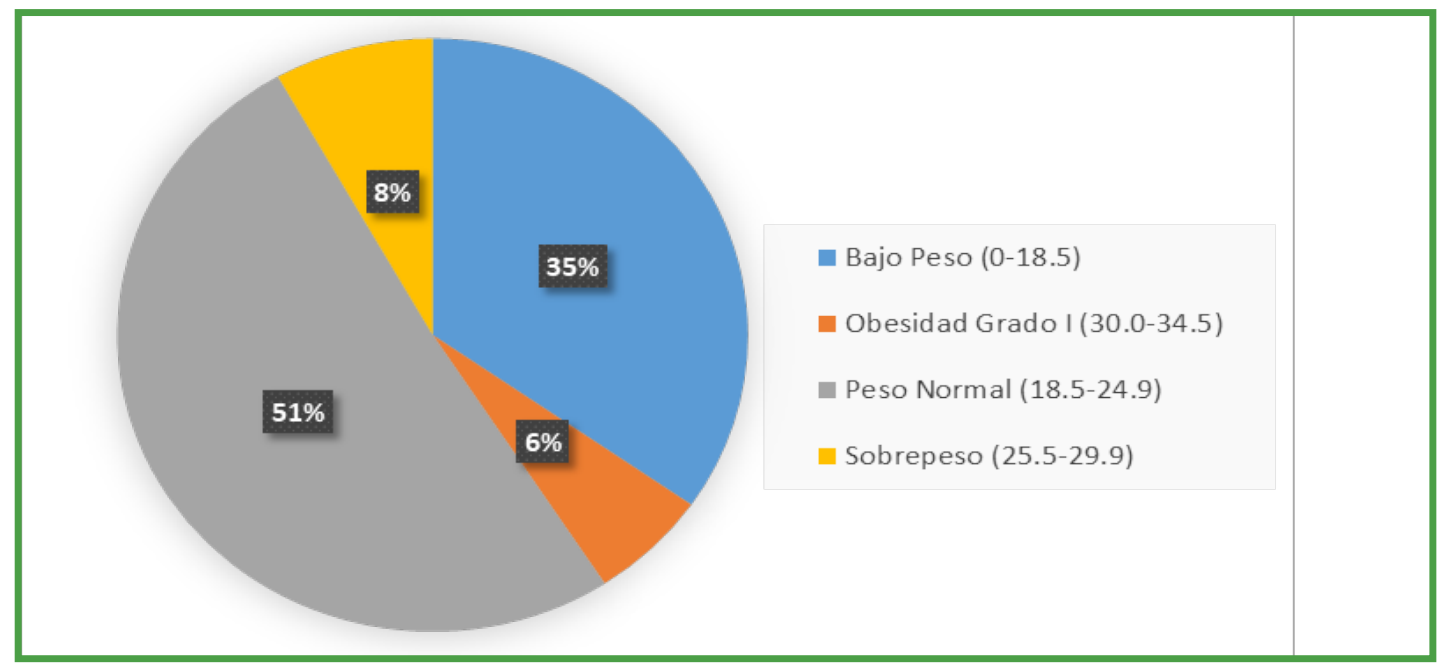

Figura 2: Porcentajes según índice de Masa corporal (IMC) en pacientes pediátricos 


\begin{tabular}{|lcc|}
\hline \multicolumn{1}{|c|}{ Tabla 1: Etiología de la DM1 en pacientes pediátricos } \\
Diagnóstico Etiológico & Frecuencia & Porcentaje \\
\hline Autoinmune & 39 & $79,59 \%$ \\
En Estudio & 5 & $10,20 \%$ \\
Idiopática & 1 & $2,04 \%$ \\
Desconocida & 4 & $8,16 \%$ \\
\hline
\end{tabular}

\begin{tabular}{|lcc|}
\hline \multicolumn{1}{|c|}{ Tabla 2: Factores predisponentes al desarrollo de DM1 } \\
Factores Predisponentes & Frecuencia & Porcentaje \\
\hline Ninguna & 37 & $75,51 \%$ \\
Obesidad & 6 & $12,24 \%$ \\
Enf. Autoinmune & 3 & $6,12 \%$ \\
Malformación Congénita o Genética & 1 & $2,04 \%$ \\
Inf. Estreptocócica & 1 & $2,04 \%$ \\
\hline
\end{tabular}

\begin{tabular}{|lcc|}
\hline \multicolumn{1}{|c|}{ Tabla 3: Frecuencia de Complicaciones de la DM1 } & \\
\hline Ninguno & Frecuencia & Porcentaje \\
Cetoacidosis & 29 & $59,18 \%$ \\
Convulsiones & 17 & $34,69 \%$ \\
Sincope & 1 & $2,04 \%$ \\
Coma Hipoglicémico & 1 & $2,04 \%$ \\
Letargia & 1 & $2,04 \%$ \\
\hline
\end{tabular}

riesgo descubierto.

La existencia de DM1 en un familiar relativo al paciente fue de 34,69\%, (Madre: 10,20\%, Padre: 6,12\%, Hermano(a): 2,04\%, Otros: 16,33\%).

La complicación más frecuente (Tabla 3 ) fue DKA con un $35 \%$ de los casos.

El 15,38\% (n:6) de los pacientes con glicemia Arriba de $90 \mathrm{mg} / \mathrm{dl}$ presentaron datos de obesidad, mientras que el 84,62\% (n: 33) de los pacientes no.

Los valores de hemoglobina glicosilada ( $\mathrm{HbA1C}$ ) arriba de 7,5 corresponde a 35 casos del total estudiado, de los cuales 57,14\% (n: 20), eran del género femenino y 42,86\% (n:15) masculino.

En relación al cuidado de su salud el 55,10\% (n:27) de los pacientes realizaban actividad física como recomendación del médico para su manejo mientras que el 44,90\% (n:22) no cumplía con esta directriz.

\begin{tabular}{c}
\hline DISCUSIÓN \\
\hline La necesidad de objetivar información \\
epidemiológica respectiva a este tema en la población
\end{tabular}

de Honduras, fue el fundamento para la elaboración de este estudio, realizado en el Hospital María de Especialidades Pediátricas; el cual se establece como el principal centro especializado para el manejo de estas patologías pediátricas de todo el país.

La problemática para adaptarse a los cambios en el infante, afecta significativamente el controlmetabólico y la calidad de vida subsiguiente, por lo que se debe proveer a los pacientes conocimientos y herramientas que les permitan lograr un autocuidado suficiente ${ }^{17}$ ya que los niveles de actividad física, el desempeño deportivo, competitivo y profesional, pueden ser realizados por personas con DM1 sin complicaciones cuando existe un adecuado manejo de su glucemia y cuidado de salud ${ }^{18}$. Por eso la importancia notable de desarrollar una educación dirigida no solo para el autocuidado, sino también centrada en producir un cambio del comportamiento ${ }^{19}$.

Al identificar que la DM1 se manifiesta en la adolescencia, se puede relacionar a los eventos hormonales y cambios psicológicos que surgen 
en esta etapa del desarrollo de la persona. Un alto porcentaje de los pacientes se presentaron con un IMC normal y llegando a presentar incluso bajo peso en número considerable. Sumado al hecho que la mayoría de los casos no presentaban obesidad al momento diagnóstico de la enfermedad. Un alto porcentaje de los pacientes contaban con un pariente relativo con obesidad que concomitaba con esta patología, esto crea mayor interés para estudios posteriores, sobre el papel que juegan los genes que están vinculados en el desarrollo y presentación de esta enfermedad, así como el papel ambiental que existe entre el sobrepeso y la manifestación de DM1 en un nivel epigenético y sus consecuencias a posterior. Según un estudio realizado en España, Madrid no existe una clara diferencia de sexos con afectación por DM1 hasta en la pubertad, en la que se identificó mayor predominio en varone ${ }^{20}$ distinto a lo obtenido en nuestro resultado.

La presencia de $\mathrm{Hb} 1 \mathrm{Ac}$ por encima de 7,5 correspondió un poco más de la mitad de todos los casos, esto refleja la representatividad y autoría de esta prueba como herramienta diagnóstica de la patología así como la utilidad que llegaría a brindar para ayuda el momento de brindar control individualizado, junto a la educación respectiva en pacientes de seguimiento. En un estudio realizado en México se encontró que los altos niveles de $\mathrm{Hb} 1 \mathrm{Al}$ c pueden relacionarse significativamente con alteraciones en el crecimiento y desarrollo, y que los pacientes más afectados se encuentran entre las edad de 9,5 años y preadolescentes ${ }^{21}$. Debido a la pobre educación respecto a este tipo de exámenes y la falta generalizada de insumos para realizarlos en distintos centros de atención de salud se desconoce generalmente el papel y la ayuda de este como factor preventivo, postergando la identificación de la patología y el tiempo certero para un adecuado abordaje médico.

Con respecto al tratamiento de los pacientes estudiados, cabe señalar que se cumplía adecuadamente con el manejo farmacológico en su mayoría, esto es alentador ya que múltiples estudios se ha encontrado que debe haber una relación directa entre el nivel de adherencia al tratamiento farmacológico y cambios en el estilo de vida para lograr un adecuado control glucémico ${ }^{22}$. Se sabe que como parte de un estilo de vida adecuado, el ejercicio físico de manera regular ha demostrado mejorar el control de la glucemia, los factores de riesgo cardiovascular, el perfil lipídico, el grado de bienestar y contribuye a la pérdida de peso, así como también produce una disminución de la $\mathrm{HbAlc}$; el más recomendado es el ejercicio aeróbico (de baja intensidad y larga duración) con una duración de 3060 minutos por lo menos cinco veces a la semana ${ }^{23}$. Lamentablemente se constató en esta investigación, que la práctica de ejercicio físico no es considerada como parte integral del tratamiento dentro de la población ya que la mayoría de los adolescentes no realizan actividad física específica, demostrando así la relevancia que se brinda al papel de la educación respecto al manejo integral de estas enfermedades hacia el paciente como a sus padres y tutores.

\section{CONCLUSIÓN}

La mayoría de los pacientes estudiados pertenecen al sexo femenino, encontrando en ellas una incidencia máxima de DM1 a los 14 años a diferencia de los varones la cual fue a los 16 años. Se comprueba la bien conocida teoría sobre la causa que predomina en la DM1, confirmando que etiológicamente es la autoinmune; a pesar de que los pacientes no presentaron factores predisponentes en su totalidad los resultados confirman que la obesidad es el más frecuente. Cabe resaltar que los pacientes debutan con los síntomas cardinales propios de esta patología, encontrando en su mayoría la polifagia en un $36,73 \%$. La diabetes es una enfermedad que desarrolla numerosas complicaciones, dentro de las cuales la que más llego a presentarse fue la DKA en un 34,69\%.

\section{AGRADECIMIENTOS}

Al Hospital De Especialidades Pediátricas María por su colaboración en el desarrollo del proyecto, brindando la información solicitada y apoyando fuertemente el proceso investigativo. A los colegas Sandy Yolibeth Urbina, Denia Mailid Acosta, Karolyn Maldonado, José Raúl Castillo y Pedro Hernandez por su apoyo en la recolección de datos para este trabajo. 


\section{REFERENCIAS}

1. Castro K, López N, Rodríguez D, Suárez J, Llerena J. Factores de riesgo y severidad de la retinopatía diabética. Rev Cub Med Mil [Internet]. 2013 jun [citado 2018 ago30]; 42(2):181-90. Disponible en: http:// scielo.sld.cu/scielo.php?script=sci_arttexte pid=S013865572013000200007 孔lng=es.

2. Ramos W, López T, Revilla L, More L, Huamaní M, Pozo M. Resultados de la vigilancia epidemiológica de diabetes mellitus en hospitales notificantes del Perú, 2012. Rev perú med exp. Salud Pública [Internet]. 2014 ene [citado 2017 marzo 26]; 31(1): 09-15. Disponible en: $\quad h t t p: / / w w w . s c i e l o . o r g . p e / s c i e l o . p h p ? p i d=S 1726-$ $46342014000100002 \mathrm{w}^{\prime}$ script $=$ sci_arttexte'tlng=en

3. Zhang-Xu A, Vivanco M, Zapata F, Málaga G, Loza C. Actividad física global de pacientes con factores de riesgo cardiovascular aplicando el "International PhysicalActivityQuestionaire" (IPAQ). Rev Med Hered [Internet]. 2011 jul [citado 2017 marzo 26]; 22(3): 115-120. Disponible en: http://www.scielo. org.pe/scielo.php?script $=$ sci_arttextépid $=S 1018$ $130 \times 2011000300005$ blng=es.

4. GuíaSalud. Guía de Práctica Clínica sobre Diabetes. Versión completa. Introducción. [Internet]. Guiasalud. es.2010 [Citado el 26 de marzo de 2017]. Disponible en: http://www.guiasalud.es/egpc/diabetes_tipol/completa/ apartado04/definicion.html

5. Lopez J, Pino A. Diabetes mellitus tipo1. Australasian Medical Journal [Internet]. 2008;6(9):466475,10. [Citado el 26 de marzo de 2017]. Disponible en: http://apps.elsevier.es/watermark/ ctl_servlet?_f $=10$ w pident_articulo $=80000003$ ఓ pident_ usuario $=0$ ' pcontactid $=$ es pident

6. Licea M, González T. Estrategias para la prevención de la diabetes mellitus tipo 1. Rev Cubana Salud Pública [Internet].2013 dic [citado2017marzo26];39(4):733-751. Disponible en: http://scielo.sld.cu/scielo.php?script=sci arttext\&pid=S086434662013000400010\&lng=es.

7. Tébar F, Escobar F. La diabetes mellitus en la práctica clínica. 1st ed. Madrid, España: Médica Panamericana; 2009: 55-57

8. Aguirre, M, Rojas, J, Cano, R, Villalobos, M, Berrueta, L. Diabetes mellitus tipo 1 y factores ambientales: La gran emboscada. Unidad de Endocrinología Instituto Autónomo Hospital Universitario de los Andes Instituto de Inmunología Clínica. Universidad de los Andes Mérida, Venezuela. 2012; 10(3): 122134. [Citado el 26 de marzo de 2017]. Disponible en: $\quad h t t p: / / w w w . s c i e l o . o r g . v e /$ scielo.php?script $=$ sci arttexte pid $=$ S1690-31102012000300002

9. Chillarón J, Flores-Le-Roux J, Sales M, Puig-de-Dou J, Castells I, Benaiges D et al. Prevalencia y factores asociados con la presencia de albuminuria y sus estadios en los pacientes con diabetes mellitus tipo 1. Nefrología (Madrid) [Internet]. 2013 [citado 2017 marzo 26]; 33(2): 231-236. Disponible en: http:// scielo.isciii.es/scielo.php?script=sci_arttexte $p i d=S 0211$ 69952013000200011 w'lng=es.

10. Gómez-Rico I, Pérez-Marín M, Montoya-Castilla I. Diabetes mellitus tipo 1: breve revisión de los principales factores psicológicos asociados [Internet]. 10.1016/j.anpedi.2014.04.003 [Citado el 26 de marzo de 2017]. Disponible en: $h t t p: / / w w w$ analesdepediatria. org/es/diabetes-mellitus-tipo-1-breve/articulo/ S1695403314001799/

11.Montilla-PérezM,Mena-LópezN,LópezA.Efectividad de la educación diabetológica sistematizada en niños que debutan con Diabetes Mellitus tipo 1 [Internet]. 2012 [Citado el 26 de marzo de 2017]; 21(1-2): 18-22. Disponible en: http://scielo.isciii.es/scielo.php?pid=S113212962012000100005 'script $=s c i$ arttexte' $t \operatorname{lng}=p t$

12. Hipoglucemia. American Diabetes Association, 2451 Crystal Drive, Suite 900 Arlington, VA22202 [Internet]. 2013 [Citado el 26 de marzo de 2017]. Disponible en: http://www.diabetes.org/es/vivir-con-diabetes/ tratamiento-y-cuidado/el-control-de-la-glucosa-en-la-sangre/ hipoglucemia.html?referrer=https://www.google.hn/\#sthash . V6jSO1zQ.dpuf

13. Complicaciones | Guía Diabetes tipo 1. Centro para la Innovación de la Diabetes Infantil, Hospital [Internet]. 2014 [Citado el 26 de marzo de 2017]. Disponible en: https://www.diabetes-cidi.org/es/diabetes-tipo-1/debut/ complicaciones

14. Machado K, Freire M, Pérez M, Montano A. Complicaciones microvasculares en niños con diabetes mellitus tipo I. Arch-Pediatr-Urug. [Internet]. 2013 [citado 2017 Sep 10]; 84(Suppl 1): 34-41. Disponible en: $\quad h t t p: / / w w w . s c i e l o . e d u . u y /$ scielo.php?script $=s c i$ arttextepid $=$ S1688-12492013000500005 $\mathrm{s}$ lng=es.

15. Fajardo-Matarrita A, Gutiérrez-Oviedo S. Hemoglobina glicosilada como elemento pronóstico en las complicaciones macrovasculares de la diabetes mellitus [Internet]. revistas.ucr.ac.cr. 2012 [Citado el 26 de marzo de 2017]; 22 (20): 1409-4568. Disponible en: http://revistas.ucr.ac.cr/index.php/enfermeria/article/ view $/ 3558$

16. Millán, C. Las otras complicaciones de la diabetes mellitus. Unidad de Gestión Clínica de Pozoblanco Córdoba. 2014 [Online] Diabetes Práctica 05(03):97144. 2014. [Citado el 26 de marzo de 2017]. Disponible en: http://www.diabetespractica.com/files/docs/ publicaciones/141872889002_Editorial_5-3.pdf

17. Gómez-Rico I, Pérez-Marín M, Montoya-Castilla I. Diabetes mellitus tipo 1: breve revisión de los principales factores psicológicos asociados [Internet]. sciencedirect.com. 2014 [Citado el 26 de marzo de 2017]; 82(1):143-146. Disponible en: http://www.sciencedirect. com/science/article/pii/S1695403314001799

18. Vista de Guía de Práctica Clínica (GPC) para el diagnóstico, tratamiento y seguimiento de la diabetes mellitus tipo 1 en la población mayor de 15 años [Internet]. Revistaendocrino.org. 2016 [Citado el 26 de marzo de 2017]; 3(2). Disponible en: http:// revistaendocrino.org/index.php/rcedm/article/view/29/49

19. Hernandez-Rodriguez J, Licea-Puig M. Papel del ejercicio físico en las personas con diabetes mellitus [Internet]. scielo.sld.cu. 2010 [Citado el 26 de marzo de 2017]; 21(2)182-201. Disponible en: http://scielo.sld.cu/ pdf/end/v21n2/end06210.pdf

20. Barrio Castellanos R. Actualización de la diabetes tipo 1 en la edad pediátrica. En: AEPap (ed.). Curso de Actualización Pediatría 2016. Madrid: Lúa Ediciones 3.0; 2016. p. 369-77.

21. Zurita J, Dosta G, Villasís K, Rivera H, Garrido M, 
Nishimura E. Pacientes pediátricos con diabetes tipo 1: crecimiento y factores asociados con su alteración. Bol. Med. Hosp. Infant. Mex. [revista en la Internet]. 2016 Jun [citado 2019 Jul 08] ; 73( 3 ): 174-180. Disponible en: $\quad h t t p: / / w w w . s c i e l o . o r g . m x /$ scielo.php?script $=s c i$

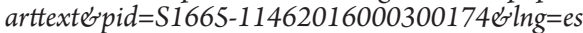

22. Alba L, Bastidas C, Vivas J, Gil F. Prevalencia de control glucémico y factores relacionados en pacientes con diabetes mellitus tipo 2 del Hospital Universitario de San Ignacio, Bogotá, Colombia [Internet]. mediagraphic.com. 2009 [Citado el 26 de marzo de 2017]; 145(6). Disponible en: http://www.medigraphic.com/pdfs/ gaceta/gm-2009/gm096a.pdf
23. Guías Clínicas Diabetes Mellitus. Guías Clínicas Semergen [Internet]. 2015 [Citado el 5 de septiembre 2017]; 91(3), 210-817. Available from: http://2016. jornadasdiabetes.com/docs/Guia_Diabetes_Semergen.pdf 